\title{
Do endometrial lesions require removal? A retrospective study
}

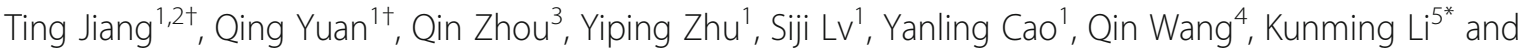
Dong Zhao ${ }^{1,6^{*}}$ (D)

\begin{abstract}
Background: This study aimed to evaluate the management of asymptomatic intrauterine lesions detected by ultrasonography.

Methods: Patients who underwent diagnostic hysteroscopy for asymptomatic lesions, including pre- and postmenopausal endometrial polyps, post-menopausal endometrial thickening ( $E T \geq 5 \mathrm{~mm}$ ) and reduplicative endometrial heterogeneity detected by transvaginal ultrasonography (TVUS), were recruited for this study.

Results: In the 792 recruited patients, the symptom-free focal masses within the uterine cavity detected by TVUS included 558 patients with pre- or post-menopausal endometrial polyps and 234 patients with postmenopausal endometrial thickening. No pre-menopausal patient presented with carcinoma. The polyp diameter (PD) was not identified as an independent risk factor for malignancy in this study. A significant difference $(P=0.036,<0.05)$ in both benign and malignant endometrial lesions was observed between two groups of post-menopausal women stratified using an endometrial thickness cut-off of $\geq 11 \mathrm{~mm}$.

The TVUS was highly sensitive (94\%) for pre-menopausal polyps. This technique had a specificity and positive predictive value of 84.4 and $92.7 \%$, respectively, for postmenopausal polyps. The TVUS was clearly valuable for ruling out polyps, as indicated by a negative likelihood ratio (LR-) of 0.087 .

Among postmenopausal women with endometrial thickening, the area under the receiver operating characteristic curve was $0.828(P<0.001)$. An ET cut-off value of $12.5 \mathrm{~mm}$ yielded a sensitivity of $72.7 \%$ and specificity of $86 \%$.

Conclusion: We recommend follow-up alone for women with asymptomatic uterine polyps, particularly those who are pre-menopausal. Additionally, gynaecologists should consider risk factors such as age, obesity, polycystic ovarian syndrome, and diabetes. Prospective long-term follow-up studies should be conducted after hysteroscopic polypectomy to evaluate the recurrence rate of endometrial lesions.
\end{abstract}

Keywords: Asymptomatic, Pre-menopausal, Post-menopausal, Hysteroscopy, Risk factors

\section{Background}

Endometrial carcinoma is a common type of gynaecological malignancy. Hysteroscopic investigation, rather than dilatation and curettage is a widely accepted diagnostic method for of endometrial cancer. In a recent report, only one of 35 asymptomatic post-menopausal

\footnotetext{
* Correspondence: likunming@hotmail.com; hendryz@gmail.com

†Ting Jiang and Qing Yuan contributed equally to this work.

${ }^{5}$ Center of Reproductive Medicine, Shanghai First Maternity and Infant Hospital, Tongji University School of Medicine, \#2699 of Gaoke Road (west), Pudong New District, Shanghai 200040, People's Republic of China

'Department of Gynecology, Shanghai First Maternity and Infant Hospital, Tongji University School of Medicine, \#2699 West Gaoke Road, Pudong New District, Shanghai 200040, People's Republic of China

Full list of author information is available at the end of the article
}

women with an endometrial thickness exceeding $4 \mathrm{~mm}$ presented with adenocarcinoma [1]. In a study of 268 women undergoing diagnostic hysteroscopy, Giannella reported only four cases of endometrial cancer and two cases of atypical endometrial hyperplasia [2]. Both studies reported a considerably low incidence of premalignant or malignant lesions in asymptomatic post-menopausal women. In another words, many of these women may have undergone unnecessary surgeries. Furthermore, the guidelines for asymptomatic endometrial lesions appear to be somewhat ambiguous.

According to the 2010 guideline for Asymptomatic Endometrial Thickening by the Society of Obstetricians

(c) The Author(s). 2019 Open Access This article is distributed under the terms of the Creative Commons Attribution 4.0 International License (http://creativecommons.org/licenses/by/4.0/), which permits unrestricted use, distribution, and reproduction in any medium, provided you give appropriate credit to the original author(s) and the source, provide a link to the Creative Commons license, and indicate if changes were made. The Creative Commons Public Domain Dedication waiver (http://creativecommons.org/publicdomain/zero/1.0/) applies to the data made available in this article, unless otherwise stated. 
and Gynaecologists of Canada (SOGC) [3] if a patient presents with an endometrial thickness $>11 \mathrm{~mm}$ or any other positive ultrasonographic findings, such as increased vascularity, an inhomogeneous endometrium, or particulate fluid, further investigations should be considered after accounting for various factors, such as age, obesity, diabetes, hypertension, and late menopausal age.

According to the 2012 Practice Guidelines for the Diagnosis and Management of Endometrial Polyps by the American Association of Gynaecologic Laparoscopists (AAGL) [4], conservative management is reasonable, particularly for asymptomatic small polyps. By contrast, removal for pathological assessment is only indicated for symptomatic polyps. In a study included 112 women with endometrial polyps, which were expectantly managed over a median period of 22.5 months (range, 6-136). There was no association between women's demographic characteristics or polyps' morphology and their growth rates. Spontaneous regression appeared to occur more frequently in premenopausal women $(P=$ $0.016)$ and in those who presented with abnormal uterine bleeding at diagnosis $(P=0.004)$; however, the differences did not reach statistical significance after correction for multiple comparisons. [5]

In China, a national guideline for asymptomatic endometrial lesions has long been needed. Currently, different institutions and even different physicians at the same institution might apply different criteria when determining whether to intervene surgically in a case of postmenopausal endometrial thickening (ET). An existing guideline for abnormal uterine bleeding (AUB) indicated that asymptomatic polyps with a diameter $<1 \mathrm{~cm}$ have a regression rate of $27 \%$. Patients with larger polyps, symptomatic polyps or infertility should undergo hysteroscopic polypectomy [6].

Once a susceptible endometrial polyp or a post-menopausal endometrial thickness $>5 \mathrm{~mm}$ has been identified by transvaginal ultrasonography (TVUS), the patient will likely be subjected to hysteroscopic polypectomy and/or curettage. Patients with an endometrium that remains inhomogeneous over time might also undergo surgical investigation after a period of observation. This preference for aggressive treatment might be partially attributable to the characteristically strict doctor-patient relationship in China.

To our knowledge, there is little evidence to indicate the appropriate interventions of asymptomatic intrauterine lesions. We therefore aimed to investigate this dilemma and identify a feasible standard for intervention in the Chinese population.

\section{Methods}

Patients who presented with asymptomatic lesions at Shanghai First Maternity and Infant Hospital from June
2013 to June 2015 were recruited. The study protocol was approved by the Ethical Committee of the Tongji University School of Medicine.

In addition to the medical history, each patient's endometrial thickness (ET), polyp diameter (PD) and pathological diagnosis was documented to evaluate the necessity of surgery and validity of the practice.

All subjects underwent hysteroscopy, which remains the gold-standard diagnostic technique for endometrial lesions [3]. Asymptomatic lesions included pre- or post-menopausal endometrial polyps, post-menopausal endometrial thickening (endometrial thickness $\geq 5 \mathrm{~mm}$ ) or reduplicative endometrial heterogeneity detected by TVUS. Inclusion criteria: Premenopausal endometrial insufficiency or uterine cavity occupying. On the 3-5th day of menstruation next month, review the ultrasound if there is still uneven endometrium or uterine cavity lesion; Postmenopausal endometrium $\geq 5 \mathrm{~mm}$ or uterine cavity lesion. Exclusion criteria: The growth stage patients with thicker endometrium were followed up by the clinic. Hysteroscopic treatment was required if there was abnormal uterine bleeding. And all the hysteroscopic surgery for abnormal uterine bleeding is beyond our scope of discussion.

Results were expressed as means \pm standard deviation (SD) or standard error of mean (SEM). Data were analysed by SPSS 22.0 (Chicago, IL, USA). The comparison between the two groups was performed with the chi-square test and $P<0.05$ indicates statistically significance. A multi-logistic regression analysis was applied to study the risk factors for malignancy in patients with asymptomatic endometrial lesions.

The study protocol was approved by the Ethical Committee of the Tongji University School of Medicine.

\section{Statistics}

All data were analysed using SPSS 19.0 (SPSS, Inc., Chicago, IL, USA). The chi-square test was applied to comparisons between groups, and a $P$ value $<0.05$ was considered to indicate a statistically significant difference.

\section{Results}

A total of 792 patients with symptom-free focal masses within the uterine cavity detected by TVUS were recruited; these included 558 patients with pre- or post-menopausal endometrial polyps and 234 patients with post-menopausal endometrial thickening (endometrial thickness $\geq 5 \mathrm{~mm}$ ) who underwent hysteroscopic resection and diagnostic curettage.

The ages of women with asymptomatic polyps ranged from 21 to 57 years in the pre-menopausal group and from 40 to 78 years in the post-menopausal group, with respective mean ages of $38.42 \pm 7.60$ and $60.85 \pm 5.41$ years, respectively. In the asymptomatic endometrial 
thickening group, the women's ages ranged from 46 to 81 years, with a mean of $60.94 \pm 5.88$ years (Table 1 ).

Asymptomatic endometrial lesions were associated with a morbidity rate of $86.9 \%$, as summarised in Table 2 . Of these cases, $13.1 \%$ were normal, $72.6 \%$ had polyps (405/558), 9.1\% had fibroids/adenomyomas (51/558), $1.4 \%$ had simple/complex hyperplasia (8/558), $0.5 \%$ had atypical hyperplasia (3/558) and $1.5 \%$ had carcinoma (10/558). No carcinomas were observed in the pre-menopausal group, and only two patients in that group had atypical hyperplasia. The pre- and post-menopausal groups differed significantly in the incidence of both benign and malignant endometrial lesions $(P=0.000,<0.05$, respectively).

In the pre-menopausal group, $14.9 \%(29 / 195)$ of patients with a PD $\geq 10 \mathrm{~mm}$ on TVUS were normal (Table 3). Only one case of atypical hyperplasia $(0.5 \%, 1 /$ 198) was observed among patients with a PD $\geq 10 \mathrm{~mm}$, and the incidence of this lesion type was $0.6 \%(2 / 363)$, regardless of menstrual status. There was no statistical difference between the premenopausal and postmenopausal groups $(P=1.0,>0.05)$.

Furthermore, 9.9\% (8/81) of post-menopausal patients with a PD $\geq 10 \mathrm{~mm}$ had carcinoma; among all post-menopausal subjects, the incidence rates of endometrial carcinoma and atypical hyperplasia were $4.9 \%$ $(10 / 203)$ and $0.5 \%(1 / 203)$, respectively. A chi-square test revealed no significant difference in these incidence rates between the groups divided by PD $(P=0.055,>0.05)$ (Table 3).

Asymptomatic post-menopausal subjects were divided into two groups according to endometrial thickness (Table 4). Significant differences in the incidence rates of benign and malignant endometrial lesions were observed between the two groups $(P=0.002,<0.05$, respectively). The vast majority of lesions were benign, including 98.2\% in the group with ET from 5 to $10 \mathrm{~mm}$, and $87.5 \%$ in the group with ET $\geq 11 \mathrm{~mm}$. The malignancy rates in those two groups were $1.2 \%(2 / 172)$ and $7.8 \%(5 / 64)$ respectively.

A multi-logistic regression analysis was applied to study the risk factors for malignancy in patients with

Table 1 The ages of the patients of asymptomatic endometrial lesions

\begin{tabular}{lclll}
\hline & \multicolumn{2}{l}{ Ages (years) } & \\
\cline { 2 - 4 } & Maximum & Minimum & Mean \pm SD \\
\hline Asymptomatic polyps & & 57 & $38.42 \pm 7.60$ \\
Premenopausal (355) & 21 & 78 & $60.85 \pm 5.41$ \\
aPostmenopausal (202) & 40 & & \\
Asymptomatic endometrial thickening & & \\
$\quad$ Postmenopausal (234) & 46 & 81 & $60.94 \pm 5.88$ \\
\hline amissing value: $n=1$ & & &
\end{tabular}

asymptomatic endometrial lesions. The results revealed that age and ET were correlated with malignancy, whereas other factors such as BMI, obesity, diabetes and hypertension were not correlated with malignancy.

A receiver operating characteristic curve was used to assess the prediction value of ET for endometrial carcinoma. The sensitivity was $72.7 \%$ if an ET value of 12.5 $\mathrm{mm}$ was set as a cut-off, and the specificity was $86 \%$ (left part of Fig. 1). All above data corresponded to post-menopausal women with endometrial thickening. When we considered the ET of post-menopausal women regardless of $\mathrm{PD}$, the area under the curve (AUC) was $0.662(P=0.024,<0.05)$, as shown in the right part of Fig. 1. An ET cut-off value of $10.5 \mathrm{~mm}$ yielded a sensitivity of $52.9 \%$ and specificity of $80.8 \%$.

An AUC of $0.715(P=0.008)$ was determined when using age to predict malignancy. An age cut-off value of 48.5 years yielded a sensitivity of $84.6 \%$ and specificity of $42.2 \%$ (Fig. 2).

Patients with an inhomogeneous endometrium detected by TVUS were divided into several groups according to the presence or absence of a polyp by hysteroscopic investigation (Table 5). Regardless of the menopausal status, these cases were usually identified as endometrial polyps, with prevalence rates of $67.3 \%$ (35/ $52)$ and $81.48 \%(66 / 81)$ in the pre- and post-menopausal groups, respectively, and $76.76 \%$ (109/142) overall.

The TVUS was highly sensitive (94\%) for pre-menopausal polyps. For post-menopausal polyps, it yielded a specificity of $84.4 \%$ and positive predictive value of $92.7 \%$. TVUS appears to be valuable for ruling out polyps, as indicated by a negative likelihood ratio (LR-) of 0.087.

\section{Discussion}

This study observed an overall incidence of atypical endometrial hyperplasia and carcinoma of $2.3 \%$ among asymptomatic patients (Table 2). Additionally, 142 cases of inhomogeneous endometrium were detected by TVUS, with ET values ranging between 2 and $22 \mathrm{~mm}$ (9.67 $\pm 3.88 \mathrm{~mm}$, nine missing values). Four subjects had endometrial polyps detected by hysteroscopy but not TVUS (Table 5).

Table 6 suggests that TVUS might not be a perfect screening modality. The American Cancer Society concluded that there was insufficient evidence to recommend any routine screening (TVUS or endometrial biopsy) for endometrial cancer [7]. Given the low specificities of these modalities, the screening of asymptomatic women would result in unnecessary examinations.

Saline infusion ultrasonography (SIS) can improve the accuracy of uterine lesion diagnosis; this technique has a high specificity and sensitivity of 94 and $88 \%$, respectively, according to a meta-analysis conducted in 2015 
Table 2 Final diagnosis in the pre-/post-menopausal of asymptomatic intrauterine polyp

\begin{tabular}{|c|c|c|c|c|c|c|}
\hline & \multicolumn{2}{|c|}{$\begin{array}{l}\text { Premenopausal } \\
(N=355)\end{array}$} & \multicolumn{2}{|c|}{$\begin{array}{l}\text { Postmenopausal } \\
(N=203)\end{array}$} & \multicolumn{2}{|c|}{$\begin{array}{l}\text { Total } \\
(N=558)\end{array}$} \\
\hline & $\mathrm{N}$ & $\%$ & $\mathrm{~N}$ & $\%$ & $\mathrm{~N}$ & $\%$ \\
\hline Proliferative/secretory/atrophy & 58 & 16.3 & 15 & 7.4 & 73 & 13.1 \\
\hline Polyp & 262 & 73.8 & 143 & 70.4 & 405 & 72.6 \\
\hline Fibroid & 24 & 6.8 & 27 & 13.3 & 51 & 9.1 \\
\hline Hyperplasia without atypical & 6 & 1.7 & 2 & 1.0 & 8 & 1.4 \\
\hline Atypical hyperplasia & 2 & 0.6 & 1 & 0.5 & 3 & 0.5 \\
\hline Carcinoma & 0 & 0 & 10 & 4.9 & 10 & 1.8 \\
\hline Other & 3 & 0.9 & 5 & 2.5 & 8 & 1.5 \\
\hline
\end{tabular}

[8]. However, this procedure has not been widely adopted as a routine evaluation in China because of its time-consuming nature. Although hysteroscopy is currently considered the gold standard [9], the potential value of SIS should be considered, as this procedure is painless, cost-effective and minimally invasive [10-12]. SIS provides an excellent diagnostic technique to diagnose the size and the anatomic location of endometrial polyps.

Hysteroscopy enables the direct visualisation of the endometrial cavity while simultaneously providing the opportunity to remove lesions such as polyps and submucosal fibroids. A recent systematic review and meta-analysis estimated the overall success rate of this procedure to be $96.9 \%$ [13].

Nevertheless, hysteroscopy has a false negative rate of $3 \%$; therefore, the endometrium should be sampled even if the uterine cavity appears to be normal [14]. In addition, hysteroscopy is invasive and expensive compared to SIS [15].

Unnecessary hysteroscopy could be considerably reduced without compromising the sensitivity for detecting malignant disease if the criterion for surgical intervention was set at an ET of $\geq 12.5 \mathrm{~mm}$ (Table 4).

In this study of patients with polyps, 40.7\% (227/558) of all subjects and $35.8 \%$ (127/355) of pre-menopausal subjects had not been given the option of observation. However, hysteroscopy detected only two patients with atypical hyperplasia in the pre-menopausal group. All biopsies of the 127 pre-menopausal women who underwent hysteroscopy without observation yielded benign results. Our data suggest that immediate hysteroscopy might not be the best option for women with polyps and no fertility issues. Rather, observation could reasonably reduce over-treatment.

A recently published meta-analysis of 10,572 pre- and post-menopausal women reported a prevalence rate of $3.57 \%$ for atypical endometrial hyperplasia and carcinoma [16], which is comparable to the findings from our study.

Recurrence rate of EPs after resection is unknown. The significantly increased incidence of colorectal polyps in cohorts that also had EPs might indicate that patients with EPs should be also referred for colonoscopy. EPs have the lowest incidence of malignant transformation as compared to colon, urinary bladder, oropharyngeal, nasal and laryngeal carcinomas [17].

A total of 188 patients were included in a study [18]. The most common histopathological results were endometrial polyp, atrophic endometrium, and surface epithelium (26.6, 22.3, and 12.8\%, respectively). None of the 57 patients without vaginal bleeding had endometrial

Table 3 The diagnosis of the polyp in pre-/post-menopausal groups divided by diameter

\begin{tabular}{|c|c|c|c|c|c|c|c|c|c|c|c|c|}
\hline & \multicolumn{6}{|c|}{ Premenopausal } & \multicolumn{6}{|c|}{ Postmenopausal } \\
\hline & \multicolumn{2}{|c|}{$\begin{array}{l}\text { Diameter } \\
>=10 \mathrm{~mm} \\
(N=195)\end{array}$} & \multicolumn{2}{|c|}{$\begin{array}{l}\text { Diameter } \\
<10 \mathrm{~mm} \\
(N=150)\end{array}$} & \multicolumn{2}{|c|}{$\begin{array}{l}\text { Total } \\
(N=363)\end{array}$} & \multicolumn{2}{|c|}{$\begin{array}{l}\text { Diameter } \\
>=10 \mathrm{~mm} \\
(N=81)\end{array}$} & \multicolumn{2}{|c|}{$\begin{array}{l}\text { Diameter } \\
<10 \mathrm{~mm} \\
(\mathrm{~N}=113)\end{array}$} & \multicolumn{2}{|c|}{$\begin{array}{l}\text { Total } \\
(N=203)\end{array}$} \\
\hline & $\mathrm{N}$ & $\%$ & $\mathrm{~N}$ & $\%$ & $\mathrm{~N}$ & $\%$ & $\mathrm{~N}$ & $\%$ & N & $\%$ & $\mathrm{~N}$ & $\%$ \\
\hline Proliferative/secret-ory/atrophy & 29 & 14.9 & 26 & 17.3 & 58 & 16.3 & 2 & 2.5 & 13 & 11.5 & 15 & 7.4 \\
\hline Polyp & 144 & 73.8 & 114 & 76 & 262 & 73.8 & 52 & 64.2 & 84 & 74.3 & 143 & 70.4 \\
\hline Fibroid & 18 & 9.2 & 3 & 2.0 & 24 & 6.8 & 14 & 17.3 & 11 & 9.7 & 27 & 13.3 \\
\hline Hyperplasia without atypical & 2 & 1.0 & 4 & 2.7 & 6 & 1.7 & 2 & 2.5 & 0 & 0 & 2 & 1.0 \\
\hline Atypical hyperplasia & 1 & 0.5 & 1 & 0.7 & 2 & 0.6 & 0 & 0 & 1 & 0.9 & 1 & 0.5 \\
\hline Carcinoma & 0 & 0 & 0 & 0 & 0 & 0 & 8 & 9.9 & 2 & 1.8 & 10 & 4.9 \\
\hline Other & 1 & 0.5 & 2 & 1.4 & 3 & 0.9 & 3 & 3.7 & 2 & 1.8 & 5 & 2.5 \\
\hline
\end{tabular}


Table 4 The diagnosis of asymptomatic endometrial thickening divided by ET

\begin{tabular}{|c|c|c|c|c|}
\hline & \multicolumn{2}{|c|}{$\begin{array}{l}E T>=5 \mathrm{~mm} \\
(N=234)\end{array}$} & \multicolumn{2}{|c|}{$\begin{array}{l}E T>=11 \mathrm{~mm} \\
(N=62)\end{array}$} \\
\hline & $\mathrm{N}$ & $\%$ & $\mathrm{~N}$ & $\%$ \\
\hline Proliferative/secretory/atrophy & 71 & 30.3 & 11 & 17.7 \\
\hline Polyp & 134 & 57.3 & 39 & 62.9 \\
\hline Fibroid & 12 & 5.1 & 2 & 3.2 \\
\hline Hyperplasia without atypical & 3 & 1.3 & 1 & 1.6 \\
\hline Atypical hyperplasia & 4 & 1.7 & 3 & 4.8 \\
\hline Carcinoma & 7 & 3.0 & 5 & 8.1 \\
\hline Other & 3 & 1.2 & 1 & 1.6 \\
\hline
\end{tabular}

cancer. This conclusion is consistent with our research. But it was not determine the cut-off value. And it point that the presence of vaginal bleeding was significantly associated with the diagnosis of endometrial cancer and any endometrial disorder $(p=0.001$ and $p=0.000$, respectively).

Our research report no significant difference in these incidence rates between the groups divided by PD $(P=0.055$, $>0.05$ ) in post-menopausal patients' endometrial carcinoma and atypical hyperplasia. Since the $P$ value is close to 0.05 , the post-menopausal patient with $P D \geq 10 \mathrm{~mm}$ should undergo the operation in our clinical recommendation.

Our analysis suggested that ET and age were risk factors for endometrial malignancy. Among post-menopausal women, an ET cut-off value of $12.5 \mathrm{~mm}$ was determined. Our data suggest that PD does not correlate with malignancy. Although ET was identified as a main factor associated with malignancy, the effectiveness of this assessment appeared to decrease. Certainly, morbidity would increase with aging. However, an age cut-off of 48.5 years could not effectively predict malignant disease in asymptomatic women.

The true incidence of asymptomatic polyps remains to be clarified [19-21]. In a study of 1870 subjects, 653 patients had been diagnosed with polyps, 117 were asymptomatic and none of the latter had carcinoma [22]. Another study of 1155 women reported that only one case of endometrial carcinoma was observed among patients with asymptomatic polyps and concluded that hysteroscopic polypectomy could be performed except in cases of polyps with large diameters [23].

The prevalence of polyp among women who underwent diagnostic hysteroscopy and blind polypectomy was more common in the age group of 40-49 years. Polyps manifested as AUB in $45.6 \%$ of our study population. The mean size of the polyp was not significantly different between premenopausal and postmenopausal women and single and multiple polyps. Histopathological study of the polyp showed two malignant polyps in our study population. Premalignant lesions i.e., endometrial hyperplasia without atypia and with atypia was found in 33 women. There was one uterine perforation, one cervical tear; one false passage and one patient had mild bleeding after the procedure. In our study, in the mean follow-up period of $37.57 \pm 28.12$ months, $3.9 \%$ (7 women) had recurrence. In the follow-up period of $16.56 \pm$
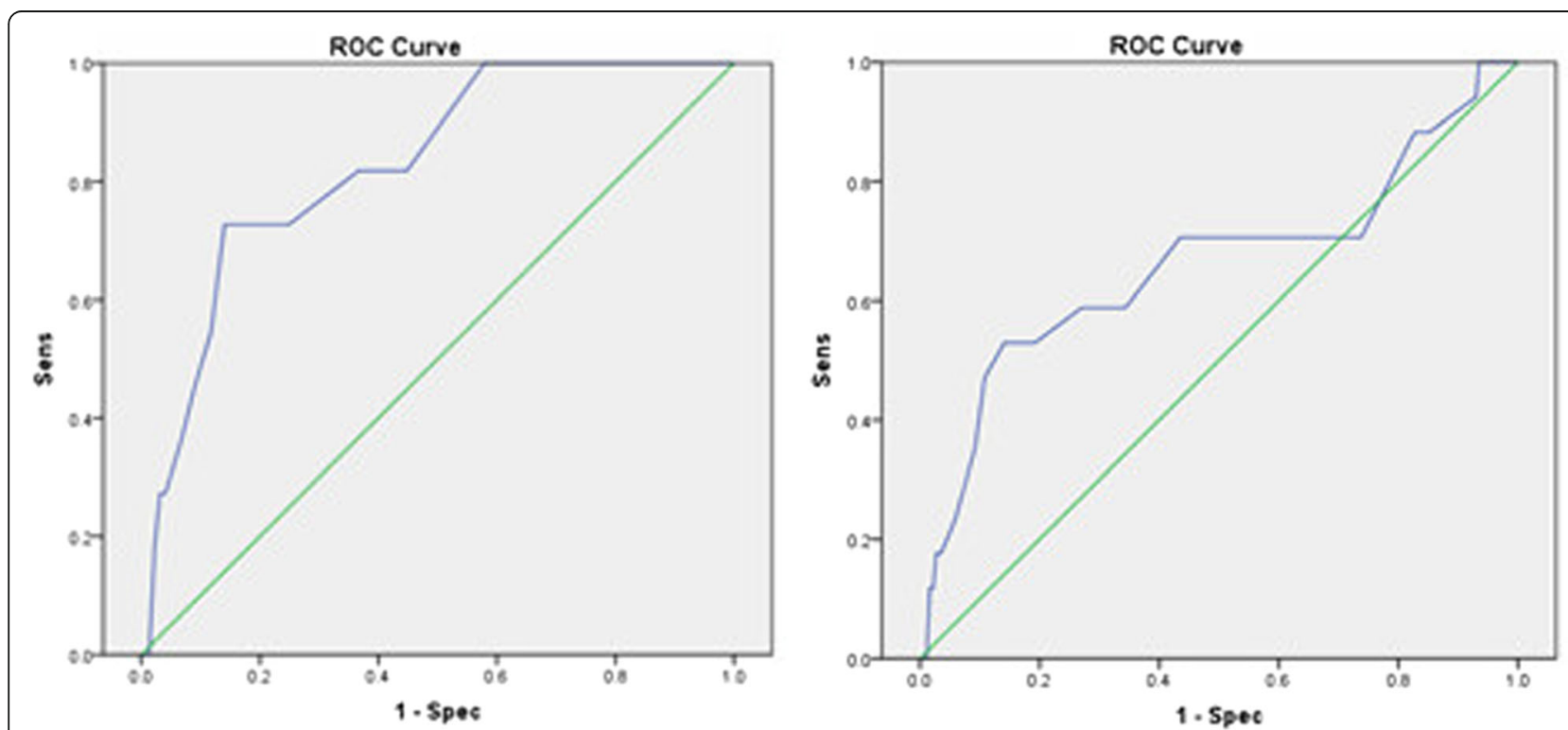

Fig. 1 Receiver operating characteristic (ROC) curve of endometrial thickness (ET) for the assessment of endometrial carcinoma. At left, postmenopausal women with endometrial thickening ( $N=234$, two missing values): the area under the ROC curve (AUC) was $0.828(P<0.001)$. An ET cut-off value yielded a sensitivity of $72.7 \%$ and specificity of $86 \%$. At right, post-menopausal women with endometrial thickening, regardless of polyp status ( $N=436,49$ missing values): the AUC was $0.662(P=0.024,<0.05)$. An ET cut-off value of $10.5 \mathrm{~mm}$ yielded a sensitivity of $52.9 \%$ and specificity of $80.8 \%$ 


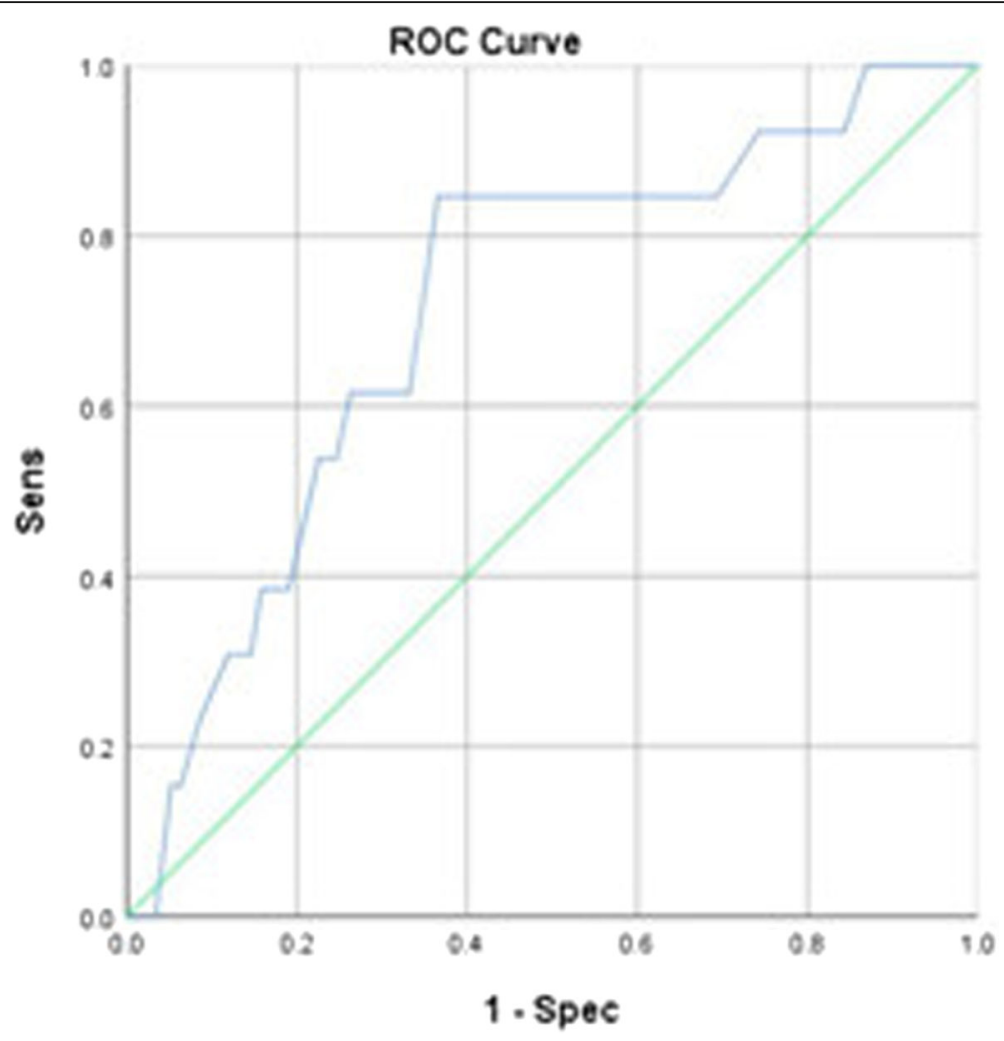

Fig. 2 The use of age to predict malignancy yielded an area under the receiver operating characteristic curve of 0.715 (P=0.008). An age cut-off value of 48.5 years yielded a sensitivity of $84.6 \%$ and specificity of $42.2 \%$

18.96 months, $78.9 \%$ women didn't have recurrence [24]. It is comparable to the findings from our study.

It remains uncertain whether the PD of an asymptomatic intrauterine lesion is a critical factor in the surgical indication. In this study, we did not find that the PD was an independent risk factor for malignancy in either preor post-menopausal women.

\section{Conclusion}

Asymptomatic polyps and ET are common gynaecological conditions. However, unnecessary interventions are administered in practice. Therefore, the elimination of over-treatment would be ideal. We conclude that asymptomatic patients with polyps, particularly those who are pre-menopausal, likely do not require immediate intervention.

Criteria for surgical intervention, such as an ET $\geq 12.5$ $\mathrm{mm}$ could potentially reduce the risk of over-treatment. In asymptomatic young woman with small EPs $<10 \mathrm{~mm}$ in size, conservative management can be safely followed by monitoring the polyp growth. EPs located at the fundal and tubocornual regions mechanically affect fertility

Table 5 Inhomogenous endometrial showed by ultrasonography, which divided by whether polyp or polyp-free found by hysteroscopy in the uterine cavity

\begin{tabular}{|c|c|c|c|c|c|c|c|c|c|c|c|c|}
\hline & \multicolumn{4}{|c|}{$\begin{array}{l}\text { Premenopausal } \\
(N=52)\end{array}$} & \multicolumn{4}{|c|}{$\begin{array}{l}\text { Postmenopausal } \\
(N=81)\end{array}$} & \multicolumn{4}{|c|}{$\begin{array}{l}\text { Total } \\
(N=142)\end{array}$} \\
\hline & \multicolumn{2}{|c|}{$\begin{array}{l}\text { Polyp } \\
(N=35)\end{array}$} & \multicolumn{2}{|c|}{$\begin{array}{l}\text { Free-polyp } \\
(N=17)\end{array}$} & \multicolumn{2}{|c|}{$\begin{array}{l}\text { Polyp } \\
(N=66)\end{array}$} & \multicolumn{2}{|c|}{$\begin{array}{l}\text { Free-polyp } \\
(N=15)\end{array}$} & \multicolumn{2}{|c|}{$\begin{array}{l}\text { Polyp } \\
(N=109)\end{array}$} & \multicolumn{2}{|c|}{$\begin{array}{l}\text { Free-polyp } \\
(N=33)\end{array}$} \\
\hline & $\mathrm{N}$ & $\%$ & $N$ & $\%$ & $\mathrm{~N}$ & $\%$ & $\mathrm{~N}$ & $\%$ & N & $\%$ & $N$ & $\%$ \\
\hline Proliferative/secretory/atrophy & 9 & 25.7 & 11 & 64.7 & 2 & 3.0 & 10 & 66.7 & 13 & 11.9 & 22 & 66.7 \\
\hline Polyp & 21 & 60.0 & 2 & 11.8 & 54 & 81.8 & 2 & 13.3 & 80 & 73.4 & 4 & 12.1 \\
\hline Fibroid & 2 & 5.7 & 0 & 0 & 6 & 9.1 & 0 & 0 & 8 & 7.3 & 0 & 0 \\
\hline Hyperplasia without atypical & 3 & 8.6 & 3 & 17.7 & 0 & 0 & 2 & 13.4 & 4 & 3.7 & 5 & 15.2 \\
\hline Atypical hyperplasia & 0 & 0 & 0 & 0 & 2 & 3.0 & 0 & 0 & 2 & 1.8 & 0 & 0 \\
\hline Carcinoma & 0 & 0 & 1 & 5.9 & 2 & 3.0 & 1 & 6.7 & 2 & 1.8 & 2 & 6.1 \\
\hline
\end{tabular}


Table 6 TVUS for the diagnosis of intrauterine pathology ${ }^{\mathrm{a}}$ in pre- and post-menopausal polyps

\begin{tabular}{lllllll}
\hline & Sens \% & Spec \% & PPV \% & NPV \% & LR+ & LR- \\
\hline Premenopausal polyps & 94.0 & 68.6 & 84.0 & 52.2 & 2.99 & 0.087 \\
Postmenopausal polyps & 51.8 & 84.4 & 92.7 & 31.4 & 3.32 & 0.57 \\
\hline
\end{tabular}

Sens sensitivity, Spec specificity, PPV positive predictive value, NPV negative predictive value, $L R+$ positive likelihood ratio, $L R$ - negative likelihood ratio ${ }^{a}$ Endometrial polyps, intrauterine fibroids and endometrial cancer

and disturb normal cellular function due to chronic inflammation. In cases where EPs are a cause of subfertility mechanical hysteroscopic resection is advisable.

EP Detection in either peri- or post-menopausal age, in symptomatic or asymptomatic patients' calls for meticulous hysteroscopic examination and polypectomy is mandatory. Endometrial curettage is also recommended to rule out sub clinical endometrial hyperplasia or cancer.

An endometrial histopathological examination should be considered, especially for patients with a history of vaginal bleeding.

Prospective long-term follow-up studies after hysteroscopy and polypectomy are needed to evaluate the recurrence rates of endometrial lesions.

\section{Abbreviations}

AUB: Abnormal uterine bleeding; AUC: Area under the curve; ET: Endometrial thickening; PD: Diameters of the polyps; ROC: Receiver operating characteristic; SIS: Saline infusion ultrasonography; TVUS: Transvaginal ultrasonography

\section{Acknowledgements}

Not applicable.

\section{Funding}

National Natural Science Foundation of China; Award number: 81471436\&81771548; the study was funded by Shanghai First Maternity and Infant Hospital. (Grant number 2015B08);

\section{Availability of data and materials}

The datasets used and/or analysed during the current study available from the corresponding author on reasonable request.

\section{Authors' contributions}

DZ: Study design; TJ and QY: Manuscript writing; QY, YZ, SL, YC, QW: History collection or management; QZ \& TJ: Data statistics; ZD \& KL: Manuscript editing. All authors read and approved the final manuscript.

\section{Ethics approval and consent to participate}

This article does not contain any studies with animals performed by any of the authors. All procedures performed in studies involving human participants were in accordance with the ethical standards of the Ethics Committee of Shanghai First Maternity and Infant Hospital. The study protocol was approved by the Ethical Committee of the Tongji University School of Medicine. Infant Hospital and committee reference number is KS18109. Written informed consent was obtained from all participants.

\section{Consent for publication}

Not applicable.

\section{Competing interests}

We declare that we have no financial and personal relationships with other people or organizations that can inappropriately influence our work, there is no professional or other personal interest of any nature or kind in any product, service and/or company that could be construed as influencing the position presented in, or the review of, the manuscript entitled.

\section{Publisher's Note}

Springer Nature remains neutral with regard to jurisdictional claims in published maps and institutional affiliations.

\section{Author details}

${ }^{1}$ Department of Gynecology, Shanghai First Maternity and Infant Hospital, Tongji University School of Medicine, \#2699 West Gaoke Road, Pudong New District, Shanghai 200040, People's Republic of China. ${ }^{2}$ Department of Gynecology, Obstetrics and Gynaecology Hospital of Fudan University, \#419 of Fangxie Road, Huangpu District, Shanghai 200011, People's Republic of China. ${ }^{3}$ Department of Biostatistics, School of Public Health Fudan University, 138\# Yixueyuan Road, Shanghai 200032, People's Republic of China. ${ }^{4}$ Department of Gynecology and Obstetrics, Punan Hospital of Shanghai, \#219 of Linyi Road, Pudong New District, Shanghai 200125, People's Republic of China. ${ }^{5}$ Center of Reproductive Medicine, Shanghai First Maternity and Infant Hospital, Tongji University School of Medicine, \#2699 of Gaoke Road (west), Pudong New District, Shanghai 200040, People's Republic of China. ${ }^{6}$ Department of Obstetrics and Gynecology, Shanghai Ninth People's Hospital, Shanghai Jiao Tong University School of Medicine, \#639 Zhi Zao Ju Road, Huangpu District, Shanghai 200011, People's Republic of China.

Received: 27 July 2018 Accepted: 12 April 2019

Published online: 06 May 2019

\section{References}

1. Aston B, Weaver E. Risks and benefits of hysteroscopy and endometrial sampling as a standard procedure for assessing serendipitous findings of endometrial thickening in postmenopausal women. Aust N Z J Obstet Gynaecol. 2014;54(6):597-9.

2. Giannella L, et al. Diagnostic accuracy of endometrial thickness for the detection of intra-uterine pathologies and appropriateness of performed hysteroscopies among asymptomatic postmenopausal women. Eur J Obstet Gynecol Reprod Biol. 2014;177:29-33.

3. Wolfman W, et al. Asymptomatic endometrial thickening. J Obstet Gynaecol Can. 2010;32(10):990-9.

4. American Association of Gynecologic Laparoscopists. AAGL practice report: practice guidelines for the diagnosis and management of endometrial polyps. J Minim Invasive Gynecol. 2012;19(1):3-10.

5. Wong $\mathrm{M}$, et al. The natural history of endometrial polyps. Hum Reprod. 2017;32(2):340-5.

6. Gynecological endocrinology collaboration of Chinese Society of Obstetrics and Gynecology. Diagnosis and treatment of abnormal uterine bleeding. Chin J Obstet Gynecol. 2014;39(11):801-6.

7. Vuento $\mathrm{MH}$, et al. Screening for endometrial cancer in asymptomatic postmenopausal women with conventional and colour Doppler sonography. Br J Obstet Gynaecol. 1999;106(1):14-20.

8. Seshadri S, et al. Diagnostic accuracy of saline infusion sonography in the evaluation of uterine cavity abnormalities prior to assisted reproductive techniques: a systematic review and meta-analyses. Hum Reprod Update. 2015;21(2):262-74.

9. Bingol B, et al. Comparison of diagnostic accuracy of saline infusion sonohysterography, transvaginal sonography and hysteroscopy. J Obstet Gynaecol. 2011;31(1):54-8.

10. Hajishaiha $\mathrm{M}$, et al. Transvaginal sonographic evaluation at different menstrual cycle phases in diagnosis of uterine lesions. Int J Women's Health. 2011:3:353-7.

11. Khan F, Jamaat S, Al-Jaroudi D. Saline infusion sonohysterography versus hysteroscopy for uterine cavity evaluation. Ann Saudi Med. 2011;31(4):387-92.

12. Almog B, et al. Saline instillation sonohysterography test after normal baseline transvaginal sonography results in infertility patients. Is it justified? Gynecol Endocrinol. 2011;27(4):286-9.

13. van Dongen $\mathrm{H}$, et al. Diagnostic hysteroscopy in abnormal uterine bleeding a systematic review and meta-analysis. BJOG. 2007:114(6):664-75.

14. Smith-Bindman R, Weiss E, Feldstein V. How thick is too thick? When endometrial thickness should prompt biopsy in postmenopausal women without vaginal bleeding. Ultrasound Obstet Gynecol. 2004;24(5):558-65. 
15. Gupta JK, et al. Ultrasonographic endometrial thickness for diagnosing endometrial pathology in women with postmenopausal bleeding: a metaanalysis. Acta Obstet Gynecol Scand. 2002;81(9):799-816.

16. Lee SC, et al. The oncogenic potential of endometrial polyps: a systematic review and meta-analysis. Obstet Gynecol. 2010;116(5):1197-205.

17. Tanos $\mathrm{V}$, et al. The management of polyps in female reproductive organs. Int J Surg. 2017;43:7-16.

18. Gunakan $\mathrm{E}$, et al. Endometrial histopathology results and evaluation of endometrial cancer risk in geriatric women. Prz Menopauzalny. 2018;17(1):18-21.

19. Fay TN, Khanem N, Hosking D. Out-patient hysteroscopy in asymptomatic postmenopausal women. Climacteric. 1999;2(4):263-7.

20. de Ziegler D. Contrast ultrasound: a simple-to-use phase-shifting medium offers saline infusion sonography-like images. Fertil Steril. 2009:92(1):369-73.

21. Lieng $M$, et al. Prevalence, 1-year regression rate, and clinical significance of asymptomatic endometrial polyps: cross-sectional study. J Minim Invasive Gynecol. 2009;16(4):465-71.

22. Fernandez-Parra J, et al. Hysteroscopic evaluation of endometrial polyps. Int J Gynaecol Obstet. 2006;95(2):144-8.

23. Ferrazzi $\mathrm{E}$, et al. How often are endometrial polyps malignant in asymptomatic postmenopausal women? A multicenter study. Am J Obstet Gynecol. 2009;200(3):235 e1-6.

24. Kanthi JM, et al. Clinical study of endometrial polyp and role of diagnostic hysteroscopy and blind avulsion of polyp. J Clin Diagn Res. 2016;10(6): Qc01-4.

Ready to submit your research? Choose BMC and benefit from:

- fast, convenient online submission

- thorough peer review by experienced researchers in your field

- rapid publication on acceptance

- support for research data, including large and complex data types

- gold Open Access which fosters wider collaboration and increased citations

- maximum visibility for your research: over $100 \mathrm{M}$ website views per year

At $\mathrm{BMC}$, research is always in progress.

Learn more biomedcentral.com/submissions 